\title{
Peritoneal dialysis-related peritonitis caused by Gordonia bronchialis: first pediatric report
}

\author{
Valentina Bruno ${ }^{1,2}$ - James Tjon ${ }^{3} \cdot$ Sandy Lin ${ }^{4} \cdot$ Helen Groves $^{2,5} \cdot$ Kescha Kazmi $^{2,5} \cdot$ Michael Zappitelli $^{1,2}$. \\ Elizabeth Harvey ${ }^{1,2}$ (1)
}

Received: 5 June 2021 / Revised: 21 September 2021 / Accepted: 21 September 2021 / Published online: 11 October 2021

(c) The Author(s), under exclusive licence to International Pediatric Nephrology Association 2021, corrected publication 2021

\begin{abstract}
Introduction Gordonia species, aerobic, weakly acid-fast, Gram-positive bacilli, are a rare cause of peritonitis in patients undergoing peritoneal dialysis (PD). We report the first pediatric case of PD-related peritonitis caused by Gordonia bronchialis.

Case presentation A 13-year-old girl with chronic kidney disease (CKD) stage 5D, on continuous cycling PD (CCPD) for 8 years, presented with cloudy PD effluent, with no abdominal discomfort or fever. Intra-peritoneal (IP) loading doses of vancomycin and ceftazidime were started at home after obtaining a PD effluent sample, which showed WBC 2,340 $\times$ $10^{6} / \mathrm{L}$ (59\% neutrophils) and Gram-positive bacilli. On admission, she was clinically well and afebrile, with no history of methicillin-resistant Staphylococcus aureus (MRSA) infection, so vancomycin was discontinued, and IP ceftazidime and cefazolin were started, following a loading dose of intravenous cefazolin. Gordonia species grew after 5 days of incubation and later identified as Gordonia bronchialis. IP vancomycin was restarted as monotherapy, empirically for a total of 3 weeks therapy. A 2-week course of oral ciprofloxacin was added, based on susceptibility testing. PD catheter replacement was advised due to the risk of recurrence but was refused. A relapse occurred 16 days after discontinuing antibiotics, successfully treated with a 2-week course of IP ceftazidime and vancomycin. The PD catheter was removed and hemodialysis initiated. She received a further 2-week course of oral ciprofloxacin and amoxicillin-clavulanate post PD catheter removal. Conclusions Gordonia bronchialis is an emerging pathogen in PD peritonitis and appears to be associated with a high risk of relapse. PD catheter replacement is strongly suggested.
\end{abstract}

Keywords Peritoneal dialysis $\cdot$ Gordonia bronchialis $\cdot$ Peritonitis $\cdot$ Child

Valentina Bruno

valentina.bruno@sickkids.ca

1 Division of Nephrology, The Hospital for Sick Children, 555 University Avenue, Toronto, ON M5G 1X8, Canada

2 Department of Paediatrics, University of Toronto, Toronto, ON, Canada

3 Department of Pharmacy, The Hospital for Sick Children, Toronto, ON, Canada

4 Department of Nursing, The Hospital for Sick Children, Toronto, ON, Canada

5 Division of Infectious Diseases, The Hospital for Sick Children, Toronto, ON, Canada

\section{Introduction}

Gordonia species are ubiquitous in the environment and rarely cause infections in humans [1,2], The most frequent infections are bacteremias associated with an intravenous catheter [3,4]. There are a few reports of PD-related peritonitis caused by Gordonia species in adults [5-7], but no PD-related infection has been reported in children.

Five Gordonia species have been recognized as possible causes of human infection: terrae, bronchialis, polyisoprenivorans, sputi, and otitidis.

The low numbers of reported infections may be explained by their slow growth requiring a longer incubation time [8]. Also, advanced molecular methods, such as 16S rRNA and secAl gene sequencing, are needed to avoid misdiagnosis with other actinomycetes $[9,10]$. Given the absence of specific guidelines 
Table 1 Antimicrobial susceptibility testing

\begin{tabular}{llll}
\hline Antibiotic & $\begin{array}{l}\text { MIC }(\mu \mathrm{g} / \mathrm{ml}) \\
\text { First peritonitis }\end{array}$ & $\begin{array}{l}\text { MIC }(\mu \mathrm{g} / \mathrm{ml}) \\
\text { Second peritonitis }\end{array}$ & $\begin{array}{l}\text { Susceptibility } \\
\text { First/second peritonitis }\end{array}$ \\
\hline Amikacin & $\leq 1$ & $\leq 1$ & Susceptible/susceptible \\
Amoxicillin/clavulanic acid & $\leq 2$ & $\leq 2$ & Susceptible/susceptible \\
Ceftriaxone & $\leq 4$ & $\leq 4$ & Susceptible/susceptible \\
Ciprofloxacin & $\leq 0.12$ & $\leq 0.12$ & Susceptible/susceptible \\
Clarithromycin & 4 & 8 & Intermediate/resistant \\
Doxycycline & 0.5 & 1 & Susceptible/susceptible \\
Imipenem & $\leq 2$ & $\leq 2$ & Susceptible/susceptible \\
Linezolid & $\leq 1$ & $\leq 1$ & Susceptible/susceptible \\
Minocycline & $\leq 1$ & 2 & Susceptible/intermediate \\
Tobramycin & $\leq 1$ & $\leq 1$ & Susceptible/susceptible \\
Moxifloxacin & - & $\leq 0.25$ & $-/$ susceptible \\
Trimethoprim/sulfamethoxazole & $\leq 0.25$ & $\leq 0.25$ & Susceptible/susceptible \\
\hline
\end{tabular}

CLSI guidelines did not include MIC breakpoints for vancomycin despite the excellent clinical response to treat this infection, reported treatments vary based on singlecenter experience and antimicrobial sensitivities.

\section{Case presentation}

A 13-year-old girl with CKD stage 5D secondary to focal segmental glomerulosclerosis, on CCPD for 8 years using a double-cuff swan neck coil PD catheter, presented to the emergency department (ED) with cloudy PD effluent and no abdominal discomfort or fever.

Her past history was relevant for a failed kidney transplant, due to EBV-related post-transplant lymphoproliferative disorder (PTLD) and BK viremia. Her PD course was complicated by two peritonitis episodes 3 years $(S$. pneumoniae) and 18 months (S. agalactiae group B) prior to this episode. She was anuric, with normal serum albumin. An immunological work-up performed 2 years previously for pre-transplant planning, including TBNK analysis and lymphocyte proliferation assay, was normal.

At home, several hours prior to presenting to hospital, a PD fluid sample was obtained, and intra-peritoneal (IP) loading doses of vancomycin $(1,000 \mathrm{mg} / \mathrm{L})$ and ceftazidime $(500 \mathrm{mg} / \mathrm{L})$ were administered. Vancomycin was used as her cefazolin supply had expired. Cefepime is not available in Canada.

The patient was admitted for peritonitis management. PD fluid cell count showed WBC $2,340 \times 10^{6} / \mathrm{L}(59 \%$ neutrophils).

As she was afebrile, with minimal abdominal pain, and no history of MRSA infection, vancomycin was discontinued, and IP maintenance doses of ceftazidime $(125 \mathrm{mg} / \mathrm{L})+$ cefazolin $(125 \mathrm{mg} / \mathrm{L})$ were started, following a loading dose of intravenous cefazolin to avoid interruption in her CCPD regimen. After $24 \mathrm{~h}$, Gram stain revealed beaded Gram-positive bacilli.

Blood culture was negative. After 5 days, the PD fluid culture grew Gordonia species, isolated from $1 \mathrm{ml}$ placed into a blood culture bottle, subsequently identified as Gordonia bronchialis by $16 \mathrm{~s}$ rRNA gene sequencing. The infectious diseases (ID) team suggested vancomycin as monotherapy empirically, based on the literature [5]. Cefazolin and ceftazidime were discontinued. She received a loading dose of IP vancomycin $(1,000 \mathrm{mg} / \mathrm{L})$, followed by IP maintenance $(25 \mathrm{mg} / \mathrm{L})$. Vancomycin levels were monitored with an initial vancomycin level of $35.5 \mathrm{mg} / \mathrm{L} \mathrm{(13} \mathrm{h}$ after the loading dose), reduced to $29.1 \mathrm{mg} / \mathrm{L} 37 \mathrm{~h}$ after the loading dose, reaching steady-state levels of $22.6 \mathrm{mg} / \mathrm{L}$ by 7 days. The patient received maintenance IP vancomycin, supplemented by IV loading doses $(10 \mathrm{mg} / \mathrm{kg})$ when the serum vancomycin levels fell below $15 \mathrm{mg} / \mathrm{L}$. IV loading doses were chosen to avoid disruption in her CCPD schedule.

The patient remained clinically well, with good PD catheter function and ultrafiltration. PD fluid culture was negative after $48 \mathrm{~h}$ of antibiotic therapy. She was discharged after 1 week, on maintenance IP vancomycin, and completed 3 weeks total therapy with vancomycin given the positive clinical response. The antimicrobial susceptibility testing using standards set by the Clinical Laboratory Standards Institute (CLSI) revealed susceptibility to multiple antibiotics (Table 1). However, CLSI guidelines do not include MIC breakpoints for vancomycin (likely due to absence of clinical data, no standard reporting method, and/or no in vivo activity). Therefore, a 2-week course of oral ciprofloxacin was added. The patient also received antifungal prophylaxis with nystatin, while on all antibiotic treatment.

Serum vancomycin levels were monitored as an outpatient and were $22.6-26.5 \mathrm{mg} / \mathrm{L}$. Audiometry was performed 
at the end of 3 weeks of therapy and 1 week later and was normal.

Removal and reinsertion of a new PD catheter was strongly recommended due to the high reported recurrence risk [5-7], but the family declined in favor of careful monitoring for recurrence. As the patient was clinically well, she was discharged on IP antibiotics, with close follow-up.

A relapse occurred 16 days after discontinuing antibiotics. The patient presented again with cloudy effluent and no fever. Peritoneal fluid WBC was increased to $433 \times 10^{6} / \mathrm{L}$ (55\% neutrophils). Empiric IP vancomycin and ceftazidime were prescribed. The Gordonia species grew again on BHI medium from the fungal culture, after 8 days of incubation.

PD fluid culture was negative after $72 \mathrm{~h}$ of antibiotic treatment. The patient received 2 weeks of IP ceftazidime and vancomycin, and the PD catheter was removed at the end of the treatment period. She received oral ciprofloxacin and amoxicillin-clavulanate for 2 weeks after catheter removal.

A tunneled central line was inserted at the time of PD catheter removal, and the patient was started on intermittent hemodialysis (HD) with the plan to insert a new PD catheter after 1 month of HD. However, the patient has chosen to remain on $\mathrm{HD}$.

\section{Discussion}

Gordonia species are ubiquitous aerobic actinomycetes and are a rare cause of infection in humans [1,2]. The reported infections caused by these species include endocarditis, pleural infection, and peritonitis [3, 4]. Gordonia species are known to be opportunistic pathogens, and infections often occur in immunocompromised patients. They were first reported by Tsukamura in 1971, who described a new genus Gordona (in honor of the bacterial systematist Ruth E. Gordon) including weakly acid-fast organisms acquired from sputa of patients with pulmonary disease and in soil [11]. This genus was then renamed Gordonia in 1997 [12].

Gordonia bronchialis are usually found in soil, sludge, prosthetic materials, and other environments. Peritoneal dialysis-related peritonitis caused by these species has been reported in the adult literature [6,7], with the first case of Gordonia bronchialis described by Sukackiene et al. in 2017 in a 32-year-old male farmer on CCPD [5]. The PD catheter was removed in 7 of the 11 reported cases, and most of them (5 of the 7) were able to resume PD successfully [7].

Microbiologic diagnosis is difficult, and they are commonly misidentified as other actinomycetes or mycobacteria. 16S rRNA and secAl gene sequencing are currently the most accurate ways to identify Gordonia species $[9,10]$. Our patient represents the first reported pediatric PD-related infection.
The 2012 International Society of Peritoneal Dialysis (ISPD) pediatric guidelines do not include any specific recommendation for treating Gordonia sp. infections [13]. Empiric IP vancomycin is the most frequent treatment reported, while the subsequent antibiotic choice is guided by in vitro susceptibility testing. The duration of therapy is unclear and should be established based on clinical response and the immunosuppression status of the patient.

In our patient, the ID team suggestion to administer IP vancomycin as monotherapy to treat the first episode was successful. The choice to add oral ciprofloxacin was related to susceptibility testing and good oral bioavailability, in the absence of susceptibility data for vancomycin, despite the excellent clinical response. Unfortunately, a relapse occurred within a few weeks, leading to PD catheter removal.

From a pathogenic perspective, the ability of Gordonia species to create a biofilm could explain the difficulty to eradicate the infection from the PD catheter. Adhesive properties are apparently due to the presence of type $B$ peptidoglycan, long aliphatic chains of the mycolic acids within the cell wall which, together with production of biosurfactants, are important factors in biofilm formation on hydrophobic surfaces to which they adhere $[8,14]$.

The source of Gordonia bronchialis infection in our patient remains unclear. Despite a remote history of PTLD, she had no evidence of immune dysfunction. She did not travel or visit a farm. However, due to the COVID-19 pandemic, her father was avoiding "delivery in person" of the PD solution, so the PD supplies were delivered to and stored in his garage. Given that Gordonia bronchialis are widespread in the environment, the infection could be related to contamination during storage.

\section{Conclusions}

PD-related peritonitis caused by Gordonia bronchialis is uncommon in the adult PD population, and no cases have been reported in children to date. Based on our experience and review of the literature, for PD-related peritonitis caused by Gordonia bronchialis, we strongly recommend PD catheter exchange, following documentation of a negative culture with the initial antibiotic regimen. Duration of therapy should be a minimum of 2 weeks and possibly longer depending on the timing of catheter exchange following initial management. As an alternative, 3 weeks of IP antibiotic treatment should be completed, and maneuvers to eliminate the biofilm, such as intraluminal catheter treatment with tissue plasminogen activator, should be considered [15].

The diagnosis of Gordonia can be challenging, as isolation from PD fluid can require a longer incubation period 
and molecular methods for confirmation. Given the difficulty in identifying this species and the absence of specific recommendations to guide the treatment, collaboration with ID, microbiology, and pharmacy is critical.

Author contribution Valentina Bruno wrote the first draft of the manuscript. All authors reviewed and edited the manuscript and approved the final version.

Data availability Not applicable.

Code availability Not applicable.

\section{Declarations}

Ethics approval The Hospital for Sick Children does not require ethical approval for reporting individual cases or case series.

Consent for publication Written informed consent for publication of their clinical details was obtained from patient's parents. A copy of the consent form is available for review by the editors of this journal.

Conflict of interest The authors declare no competing interests.

\section{References}

1. Blaschke AJ, Bender J, Byington CL, Korgenski K et al (2007) Gordonia species: emerging pathogens in pediatric patients that are identified by $16 \mathrm{~S}$ ribosomal RNA gene sequencing. Clin Infect Dis 45:483-486

2. Sowani H, Kulkarni M, Zinjarde S (2018) An insight into the ecology, diversity and adaptations of Gordonia species. Crit Rev Microbiol 44:393-413

3. Lesens O, Hansmann Y, Riegel P, Heller R et al (2000) Bacteremia and endocarditis caused by a Gordonia species in a patient with a central venous catheter. Emerging Infect Dis 6:382-385

4. Ramanan P, Deziel PJ, Wengenack NL (2013) Gordonia Bacteremia. J Clin Microbiol 51:3443-3447

5. Sukackiene D, Rimsevicius L, Kiveryte S, Marcinkeviciene K et al (2018) A case of successfully treated relapsing peritoneal dialysis-associated peritonitis caused by Gordonia bronchialis in a farmer. Nephrol Ther 14:109-111

6. Ma TKW, Chow KM, Kwan BCH, Lee KP et al (2014) Peritonealdialysis related peritonitis caused by Gordonia species: report of four cases and literature review. Nephrology (Carlton) 19:379-383

7. Lam JYW, Wu AKL, Leung WS, Cheung I et al (2015) Gordonia species as emerging causes of continuous-ambulatory-peritonealdialysis-related peritonitis identified by $16 \mathrm{~S}$ rRNA and secA1 gene sequencing and matrix-assisted laser desorption ionization-time of flight mass spectrometry (MALDI-TOF MS). J Clin Microbiol 53:671-676

8. Arenskotter M, Broker D, Steinbuchel A (2004) Biology of the metabolically diverse genus Gordonia. Appl Environ Microbiol 70:3195-3204

9. Johnson JS, Spakowicz DJ, Hong BY, Petersen LM et al (2019) Evaluation of 16S rRNA gene sequencing for species and strainlevel microbiome analysis. Nat Commun 10:5029

10. Wang T, Kong F, Chen S, Xiao M et al (2011) Improved identification of Gordonia, Rhodococcus and Tsukamurella species by 5'-end 16S rRNA gene sequencing. Pathology 43:58-63

11. Tsukamura M (1971) Proposal of a new genus, Gordona, for slightly acid-fast organisms occurring in sputa of patients with pulmonary disease and in soil. J Gen Microbiol 68:15-26

12. Stackebrandt E, Rainey FA, Ward-Rainey NL (1997) Proposal for a new hierarchic classification system, Actinobacteria classis nov. Int J Syst Bacteriol 47:479-491

13. Warady BA, Bakkaloglu S, Newland J, Cantwell M et al (2012) Consensus guidelines for the prevention and treatment of catheter-related infections and peritonitis in pediatric patients receiving peritoneal dialysis: 2012 update. Perit Dial Int 32(Suppl 2):S32-S86

14. Bendinger B, Rijnaarts HHM, Altendorf K, Zehnder AJB (1993) Physicochemical cell surface and adhesive properties of coryneform bacteria related to the presence and chain length of mycolic acids. Appl Environ Microbiol 59:3973-3977

15. McGuire AL, Bennett SC, Lansley SM, Popowicz ND et al (2015) Preclinical assessment of adjunctive tPA and DNase for peritoneal dialysis associated peritonitis. PLoS One 10:e119238

Publisher's note Springer Nature remains neutral with regard to jurisdictional claims in published maps and institutional affiliations. 